\title{
Determinación experimental y verificación por el método del elemento finito de la frecuencia natural de vibración de barras de madera
}

\author{
Experimental determination and verification by the finite element method \\ of the natural vibration frequency of wooden bars
}

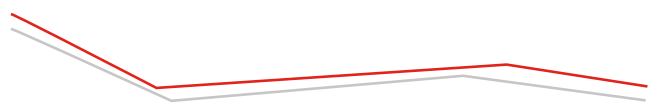

Javier Ramón Sotomayor-Castellanos*®, Víctor López-Garza**

Sotomayor-Castellanos, J. R., \& López-Garza, V. (2019). Determinación experimental y verificación por el método del elemento finito de la frecuencia natural de vibración de barras de madera. Investigación y Ciencia de la Universidad Autónoma de Aguascalientes, 27(76), 32-40.

RESUMEN

El método del elemento finito se ha utilizado de manera extensiva en la caracterización mecánica de la madera. El objetivo de la investigación fue determinar de manera experimental y verificar numéricamente la frecuencia natural en vibraciones transversales de pequeñas barras de 12 maderas. Se determinaron experimentalmente la densidad, la frecuencia de vibración y el módulo dinámico. Además, se estimó numéricamente la frecuencia correspondiente a los parámetros materiales determinados experimentalmente. El uso del módulo dinámico obtenido experimentalmente y empleado en las simulaciones conduce a valores de frecuencia equivalentes a los obtenidos en las pruebas de vibraciones, probando así la eficiencia del modelado numérico para estimar la frecuencia de una pieza de madera. Además, la correlación

Palabras clave: caracterización mecánica; densidad de la madera; módulo dinámico; vibraciones transversales; parámetros materiales; modelado numérico. Keywords: mechanical characterization; density of wood; dynamic modulus; transverse vibrations; material parameters; numerical modeling.

Recibido: 10 de marzo de 2018, aceptado: 15 de octubre de 2018

* Facultad de Ingeniería en Tecnología de la Madera, Universidad Michoacana de San Nicolás de Hidalgo. Avenida Francisco J. Múgica S/N, Ciudad Universitaria, C. P. 58030, Morelia, Michoacán, México. Correo electrónico: madera999@yahoo.com. ORCID: http://orcid. org/0000-0002-1527-8801

** Facultad de Ingeniería Mecánica, Universidad Michoacana de San Nicolás de Hidalgo. Avenida Francisco J. Múgica $S / N$, Ciudad Universitaria, C. P. 58030, Morelia, Michoacán, México. Correo electrónico: nepesh@hotmail.com. ORCID: http://orcid.org/0000-00019090-9119

Autor para correspondencia entre la frecuencia numérica y la frecuencia experimental explica $66 \%$ de esta dependencia. Así, la regresión lineal calculada indica una buena calidad predictiva del modelo de simulación empleado en la investigación.

ABSTRACT

The finite element method has been used extensively in the mechanical characterization of wood. The objective of the research was to determine experimentally and verify numerically, the natural frequency in transverse vibrations of small bars of 12 woods. The density, the frequency of vibration and the dynamic module were determined experimentally. In addition, the frequency corresponding to the material parameters determined experimentally was numerically estimated. The use of the dynamic module obtained experimentally and used on simulations, leads to frequency values equivalent to those obtained in vibration tests, thus proving the efficiency of numerical modeling to estimate the frequency of a piece of wood. In addition, the correlation between the numerical frequency and the experimental frequency explains $66 \%$ of this dependence. Thus, the calculated linear regression indicates a good predictive quality of the simulation model used in the investigation.

INTRODUCCIÓN

El desarrollo de programas computacionales permite que el modelado numérico sea más accesible en ingeniería y diseño. Sin embargo, las simulaciones requieren datos experimentales de las propiedades físicas de los materiales utilizados (Gonçalves, Trinca, 
\& Pellis, 2014). El método del elemento finito (MEF) es una técnica recomendada por ser económica y con gran potencial para el análisis de casos prácticos. No obstante, no está exento de incertidumbre y subjetividad en la interpretación de resultados (Gaff, Gašparik, Borůvka, \& Babiak, 2015).

El MEF se ha utilizado de manera extensiva en la caracterización mecánica de productos forestales (Mackerle, 2005). Entre otras aplicaciones, para profundizar el estudio de nudos en la resistencia mecánica de la madera (Baño, Arriaga, Soilán, \& Guaita, 2011; Hackspiel, De Borst, \& Lukacevic, 2014a, 2014b), para estudiar el fenómeno de ruptura mecánica de la madera (Vasic, Smith, \& Landis, 2005), el desarrollo de esfuerzos de crecimientos en árboles (Ormarsson, Dahlblom, \& Johansson, 2009), la simulación de esfuerzos asociados a la flexión de la madera (Gaff et al., 2015) y la verificación del análisis modal en la evaluación de las frecuencias de vibración en elementos estructurales de madera (Kouroussis, Ben Fekih, \& Descamps, 2017).

Por otra parte, el MEF se ha empleado para el estudio de productos de madera. Por ejemplo, Hwang y Suzuki (2016) observaron una fuerte correlación lineal $\left(R^{2}=0.99\right)$ entre las frecuencias experimentales de tambores de Zelkova serrata $\left(\rho_{\mathrm{CH}}=620 \mathrm{~kg} \mathrm{~m}^{-3}\right)$ en vibración y las frecuencias teóricas correspondientes pero determinadas con el método del elemento finito. Christoforo et al. (2016), con ayuda del MEF, determinaron el módulo de elasticidad estático en vigas la madera redonda. Por su parte, Kandler, Füssl y Eberhardsteiner (2015) relacionan diferentes enfoques estocásticos, siempre aplicando el método del elemento finito, para la caracterización de productos derivados de la madera.

El análisis modal realizado por el MEF requiere, por una parte, información sobre la configuración geométrica y las condiciones de frontera. Por otra parte, demanda los parámetros materiales densidad, módulo de elasticidad y coeficiente de Poisson (Gaff et al., 2015). Como resultado, el análisis modal proporciona los modos y las frecuencias de vibración. Por ello, la comparación experimental y numérica se enfoca en las frecuencias observadas experimentalmente durante pruebas de vibraciones y las frecuencias resultantes del MEF (García, Sampaio, \& Rosales, 2016).
En el contexto de la investigación, la madera es modelada como un sólido elástico, macroscópicamente poroso pero homogéneo, con propiedades de un medio continuo y con simetrías materiales y elásticas ortotrópicas (Guitard \& Gachet, 2004). Sin embargo, la hipótesis de ortotropía material ha sido simplificada para considerar a la madera como un material isotrópico y así estudiar toda la probeta como un volumen sólido. Así, la probeta es idealizada como una viga continua, de geometría uniforme y de masa estructuralmente homogénea. Estas hipótesis simplificadoras de la descripción de la estructura del material y del cuerpo sólido en estudio han sido propuestas entre otros autores por De Amicis, Riggio, Girardi y Piazza (2011), De Borst et al. (2013) y Hofstetter, Hellmich y Eberhardsteiner (2004).

A partir de la medición de la frecuencia de resonancia en el primer modo de vibración de probetas de madera las vibraciones transversales permiten determinar de manera no destructiva su módulo dinámico (Machek, Militz, \& Sierra-Alvarez, 2001) y viceversa, a partir del módulo dinámico es posible determinar la frecuencia natural (Kouroussis et al., 2017). Por consiguiente, si se determinan la frecuencia y el módulo de elasticidad de la madera, obtenidos de manera experimental o numérica, se podrá facilitar la caracterización dinámica de piezas de madera.

La revisión de la bibliografía revela escasa información acerca de las propiedades dinámicas de las especies estudiadas (Sotomayor-Castellanos, 2015). Por su parte, Tamarit Urias y López Torres (2007) y Silva Guzmán et al. (2010) reportan módulos de elasticidad determinados en condiciones de carga estáticas. De la misma manera, no se encontró información sobre el modelado por el método del elemento finito del comportamiento dinámico de las especies estudiadas.

El objetivo de la investigación fue determinar experimentalmente y verificar por el método del elemento finito la frecuencia natural de barras de madera ensayadas en vibraciones transversales. Para eso se experimentó con muestras de madera de 12 especies endémicas de México.

\section{MATERIALES Y MÉTODOS}

El material experimental consistió en piezas de madera aserrada recolectadas en aserraderos 
issn 1665-4412, e-issn 2521-9758

Sotomayor-Castellanos, J. R., \& López-Garza, V. del estado de Michoacán, México (longitud Oeste $101^{\circ} 49^{\prime} 59.99^{\prime \prime}$, latitud Norte 19० 10'0.01' '). Analizar un amplio rango de densidades aparentes de la madera fue el criterio para la selección de especies. La lista de especies estudiadas se presenta en la tabla 1. De cada especie se prepararon 32 probetas orientadas en las direcciones radial ( $R$ ), tangencial (T) y longitudinal (L) y con dimensiones de $0.02 \mathrm{~m}$ x $0.02 \mathrm{~m} \times 0.32 \mathrm{~m}$ (International Organization for Standardization, 2012). Las probetas se prepararon con madera sin anomalías estructurales y libre de defectos naturales. Las especies fueron identificadas en el Laboratorio de Mecánica de la Madera, de la Facultad de Ingeniería en Tecnología de la Madera, de la Universidad Michoacana de San Nicolás de Hidalgo, en Morelia, Michoacán, México.

La madera se almacenó durante 6 meses en una cámara de acondicionamiento con una temperatura de $20^{\circ} \mathrm{C}\left( \pm 1^{\circ} \mathrm{C}\right)$ y una humedad relativa de $60 \%( \pm 2 \%)$, hasta que su peso fue constante. El contenido de humedad de la madera se calculó de acuerdo con la norma ISO 13061-1:2014 (International Organization for Standardization, 2014a). La densidad aparente de la madera al momento del ensayo se calculó según la norma ISO 13061-2:2014 (International Organization for Standardization, 2014b). En lo subsecuente se escribirá densidad.

La determinación experimental del módulo dinámico siguió el procedimiento reportado por Villaseñor-Aguilar y Sotomayor-Castellanos (2015). Las pruebas de vibraciones transversales consistieron en medir la frecuencia natural de vibración perpendicular a la dirección longitudinal de la probeta. A este parámetro se le define como frecuencia experimental $\left(f_{\text {exp }}\right)$. Con tal propósito se utilizó un analizador de frecuencias Grindosonic ${ }^{\circledR}$ MK7 (Rango: $20 \mathrm{kHz}$ - $100 \mathrm{kHz}$ ). La figura 1 muestra una fotografía de las pruebas de vibraciones transversales. El impulso elástico inicial fue aplicado en el centro geométrico de la cara superior de la probeta, en la dirección transversal a la misma, apoyada sobre dos soportes rígidos de tipo simple a una distancia nodal de $0.224 \mathrm{I}$. El ensayo dinámico en cada probeta fue repetido tres veces y el promedio de valores fue considerado para su análisis posterior.

A partir de la ecuación de movimiento de una viga en vibraciones transversales (1) (Weaver, Timoshenko, \& Young, 1990):

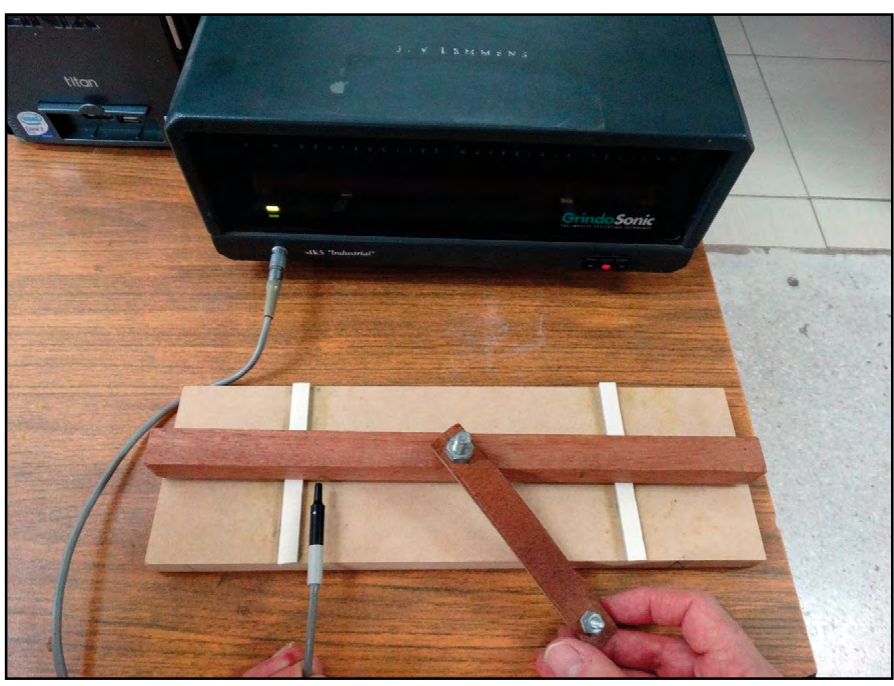

Figura 1. Prueba de vibraciones transversales.

Fotografía del equipo de investigación.

EI $\frac{\partial^{4} y}{\partial x^{4}}+m_{1} \frac{\partial^{2} y}{\partial t^{2}}-\left(m_{1} r^{2}+\frac{E l m_{1}}{K^{\prime} A G}\right) \frac{\partial^{4} y}{\partial x^{2} \partial t^{2}}+\frac{m_{1}^{2} r^{2}}{K^{\prime} A G} \frac{\partial^{4} y}{\partial t^{4}}=0$

Donde:

$E=$ Módulo de elasticidad $\left(\mathrm{N} \mathrm{m}^{-2}\right)$

$I=$ Momento de inercia de la sección transversal de la probeta $\left(\mathrm{m}^{4}\right)$

$m_{1}=$ Masa por unidad de longitud de la probeta $\left(\mathrm{kg} \mathrm{m}^{-1}\right)$

$A=$ Área de la sección transversal de la probeta $\left(\mathrm{m}^{2}\right)$

$\mathrm{G}=$ Módulo de Rigidez de la madera $\left(\mathrm{N} \mathrm{m}^{-2}\right)$

$y=$ Desplazamiento en la dirección transversal de la probeta (m)

$x=$ Distancia en la dirección longitudinal de la probeta (m)

$\dagger=$ Tiempo $(\mathrm{s})$

$\mathrm{K}^{\prime}$ = Factor de forma en cortante $(0.833$ para probetas prismáticas)

$r=$ Radio de giro de la sección transversal de la probeta $\left(m^{2}\right)$, con: $r=\sqrt{1 / A}$

El módulo dinámico se calculó con la ecuación (2) (Machek et al., 2001):

$$
E_{\text {exp }}=\frac{4 \pi^{2} L^{4} f_{\text {exp }}^{2} \rho_{C H}}{m^{4} r^{2}}\left(1+\frac{r^{2}}{\mathrm{l}^{2}} K\right)
$$

Donde:

$\mathrm{E}_{\mathrm{exp}}=$ Módulo dinámico $\left(\mathrm{N} \mathrm{m}^{-2}\right)$

$\mathrm{L}=$ Largo de la probeta $(\mathrm{m})$ 
$\mathrm{I}=\quad$ Distancia entre apoyos $(\mathrm{m})$

$f_{\text {exp }}=$ Frecuencia natural experimental de la probeta $(\mathrm{Hz})$

$\rho_{\mathrm{CH}}=$ Densidad de la madera a un contenido de humedad $\mathrm{CH}\left(\mathrm{kg} \mathrm{m}^{-3}\right)$

$\mathrm{m}, \mathrm{K}=$ Constantes adimensionales $(12,65,49,48)$

$r=\quad$ Radio de giro de la sección transversal de la probeta $\left(\mathrm{m}^{2}\right)$ con: $r=\sqrt{1 / A}$

$I=$ Momento de inercia de la sección transversal de la probeta $\left(\mathrm{m}^{4}\right)$

$A=$ Área de la sección transversal de la probeta $\left(\mathrm{m}^{2}\right)$

Los datos experimentales del contenido de humedad de la madera, de su densidad, de su módulo dinámico, así como al de las frecuencias naturales de vibración corresponden a los antecedentes de las especies reportadas por Sotomayor-Castellanos (2015). El experimento numérico consistió en introducir en el modelo los parámetros materiales: dimensiones $(R, T, L)$, densidad $(\rho c H)$ y módulo dinámico experimental $\left(E_{\exp }\right)$ para estimar la frecuencia natural de vibración del sistema en movimiento, nombrada frecuencia numérica $\left(f_{M E F}\right)$.

El cálculo por el método del elemento finito (MEF) siguió el procedimiento reportado por Sotomayor-Castellanos y López-Garza (2016). El modelado consideró los aspectos siguientes: El programa utilizado para el análisis numérico fue ANSYS® 15. Se realizó un estudio de convergencia de la malla. Se modeló la probeta como una viga compuesta de un material isotrópico. Los valores de la densidad $\left(\rho_{\mathrm{CH}}\right)$ y del módulo dinámico experimental ( $\left.E_{\exp }\right)$ empleados corresponden a los resultados experimentales presentados en la tabla 1. El coeficiente de Poisson fue 0.3, valor sugerido por Sotomayor-Castellanos y López-Garza (2016). Los principales parámetros que se utilizaron en el MEF fueron geometría 3D; Tipo de elemento: Hexaédrico SOLID186; Tamaño de malla: 0,002 (m); Número de elementos: 16700: Número de nodos: 77495 (figura 2); y Función de forma: lineal.

No obstante que el número de probetas por cada muestra fue de 32, cantidad suficiente para considerar la teoría de las grandes muestras en el análisis, se calculó a posteriori el tamaño de la muestra necesario para validar las pruebas estadísticas. El tamaño de la muestra (número de probetas) se calculó con la ecuación (3) (Gutiérrez Pulido \& De la Vara Salazar, 2012):

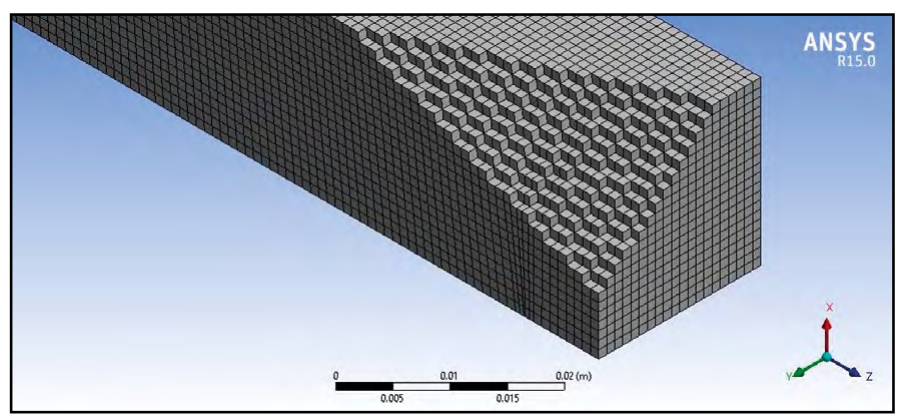

Figura 2. Mallado.

Imagen adaptada de Sotomayor-Castellanos y López-Garza (2016).

$$
n=\frac{2 \sigma^{2}}{e^{2}}
$$

Donde:

$\mathrm{n}=$ Tamaño de la muestra

$\sigma=$ Desviación estándar

e = Error de estimación aceptable

\section{Diseño experimental}

Las variables de respuesta fueron la frecuencia experimental (fexp) y la frecuencia numérica (fMEF). La densidad de la madera $\left(\rho_{\mathrm{CH}}\right)$ y el módulo dinámico (Evt) fueron variables de referencia utilizadas en el modelo numérico. El contenido de humedad de la madera $(\mathrm{CH})$ se consideró parámetro de referencia. El cálculo numérico de las frecuencias se consideró el tratamiento. Los resultados experimentales se contrastaron con los numéricos. Para esto, se calculó el estadístico media $(\bar{X})$ y se realizaron pruebas de normalidad calculando el sesgo estandarizado (S) y la curtosis estandarizada (C). El estadístico descriptivo y las pruebas de normalidad de las variables experimentales $\rho_{\mathrm{CH}}$. $E_{\mathrm{v} t}$ y fexp fueron calculados con los resultados de 32 probetas diferentes de cada especie en estudio.

Una vez confirmada la normalidad de las muestras y verificada la conformidad de varianzas se realizó un análisis de varianza de muestras independientes para un nivel de confianza de $95 \%$. Suponiendo una distribución normal con media cero $(\bar{X}=0)$ y varianza constante $\left(\sigma^{2}\right)$ e independientes entre sí, para cada prueba de diferencia de medias se verificó la hipótesis nula $\mathrm{H}_{0}: \bar{X}_{\exp }-\bar{X}_{F E M}=0$, y se contrastó con la hipótesis alterna $\mathrm{H}_{\mathrm{A}}$ : $\overline{\mathrm{X}}_{\text {exp }}-\overline{\mathrm{X}}_{\mathrm{FEM}} \neq$ 0 . Los subíndices exp y FEM representan el valor correspondiente a la frecuencia experimental $\left(f_{\exp }\right)$ y a la frecuencia numérica $\left(f_{\text {MEF }}\right)$. Se calcularon regresiones lineales $(y=a x \pm b)$ y sus coeficientes 
de determinación $\left(R^{2}\right)$ entre $f_{\exp }$ en función de $\rho_{\mathrm{cH}}, \mathrm{y}$ entre fexp en función de $f_{\text {MEF }}$.

\section{RESULTADOS}

La tabla 1 presenta los resultados de la densidad, del módulo dinámico, de la frecuencia experimental y de la frecuencia numérica de las especies en estudio. La figura 3 presenta la deformación de una probeta de Cedrela odorata en el primer modo de vibración. El contenido de humedad de la madera promedio fue de $10.8 \%$ con un coeficiente de variación de $1.1 \%$. Este parámetro se consideró constante y sin influencia en los resultados.

Tabla 1

Densidad, módulo dinámico, frecuencia experimental y frecuencia numérica

\begin{tabular}{|c|c|c|c|c|c|c|}
\hline \multicolumn{3}{|c|}{ Especie } & \multirow{2}{*}{$\begin{array}{c}\begin{array}{c}\rho_{\mathrm{CH}} \\
\left(\mathrm{kg} \mathrm{m}^{-3}\right)\end{array} \\
357\end{array}$} & \multirow{2}{*}{$\begin{array}{c}\begin{array}{c}E_{\exp } \\
\left(\mathrm{MN} / \mathrm{m}^{-2}\right)\end{array} \\
3765\end{array}$} & \multirow{2}{*}{$\begin{array}{l}f_{\text {exp }} \\
(\mathrm{Hz}) \\
655\end{array}$} & \multirow{2}{*}{$\begin{array}{l}f_{M E F} \\
(H z)\end{array}$} \\
\hline 1 & Spathodea campanulata & $\bar{x}$ & & & & \\
\hline & & $\sigma$ & 25 & 516 & 68 & - \\
\hline & & CV & 7,0 & 13,7 & 10,4 & - \\
\hline \multirow[t]{3}{*}{2} & Gyrocarpus americanus & $\bar{x}$ & 392 & 6107 & 786 & 814 \\
\hline & & $\sigma$ & 14 & 822 & 33 & - \\
\hline & & CV & 3,6 & 13,5 & 4,2 & - \\
\hline \multirow[t]{3}{*}{3} & Cupressus lindleyi & $\bar{x}$ & 440 & 10815 & 987 & 1023 \\
\hline & & $\sigma$ & 60 & 1540 & 131 & - \\
\hline & & CV & 13,6 & 14,2 & 13,3 & - \\
\hline \multirow[t]{3}{*}{4} & Cedrela odorata & $\bar{x}$ & 566 & 9795 & 873 & 858 \\
\hline & & $\sigma$ & 51 & 1262 & 89 & - \\
\hline & & $\mathrm{CV}$ & 9,0 & 12,9 & 10,2 & - \\
\hline \multirow[t]{3}{*}{5} & Tabebuia rosea & $\bar{x}$ & 628 & 9753 & 797 & 812 \\
\hline & & $\sigma$ & 34 & 907 & 42 & - \\
\hline & & CV & 5,4 & 9,3 & 5,3 & - \\
\hline \multirow[t]{3}{*}{6} & Guazuma ulmifolia & $\bar{x}$ & 730 & 8765 & 686 & 715 \\
\hline & & $\sigma$ & 46 & 1165 & 55 & - \\
\hline & & CV & 6,3 & 13,3 & 8,0 & - \\
\hline \multirow[t]{3}{*}{7} & Pinus douglasiana & $\bar{x}$ & 734 & 11805 & 931 & 827 \\
\hline & & $\sigma$ & 64 & 1421 & 46 & - \\
\hline & & CV & 8,7 & 12,0 & 4,9 & - \\
\hline \multirow[t]{3}{*}{8} & Swietenia humilis & $\bar{x}$ & 757 & 9098 & 756 & 715 \\
\hline & & $\sigma$ & 31 & 1186 & 79 & - \\
\hline & & $\mathrm{CV}$ & 4,1 & 13,0 & 10,4 & - \\
\hline \multirow[t]{3}{*}{9} & Quercus spp. & $\bar{x}$ & 841 & 13922 & 806 & 839 \\
\hline & & $\sigma$ & 29 & 1868 & 53 & - \\
\hline & & $\mathrm{CV}$ & 3,4 & 13,4 & 6,6 & - \\
\hline \multirow[t]{3}{*}{10} & Quercus scytophylla & $\bar{x}$ & 852 & 13922 & 747 & 834 \\
\hline & & $\sigma$ & 15 & 1505 & 42 & - \\
\hline & & CV & 1,8 & 10,8 & 5,6 & - \\
\hline \multirow[t]{3}{*}{11} & Platymiscium dimorphandrum & $\bar{x}$ & 866 & 14077 & 823 & 832 \\
\hline & & $\sigma$ & 33 & 1637 & 40 & - \\
\hline & & CV & 3,8 & 11,6 & 4,9 & - \\
\hline \multirow[t]{3}{*}{12} & Acacia farnesiana & $\bar{x}$ & 901 & 13965 & 946 & 812 \\
\hline & & $\sigma$ & 41 & 1539 & 92 & - \\
\hline & & CV & 4,6 & 11,0 & 9,7 & - \\
\hline
\end{tabular}




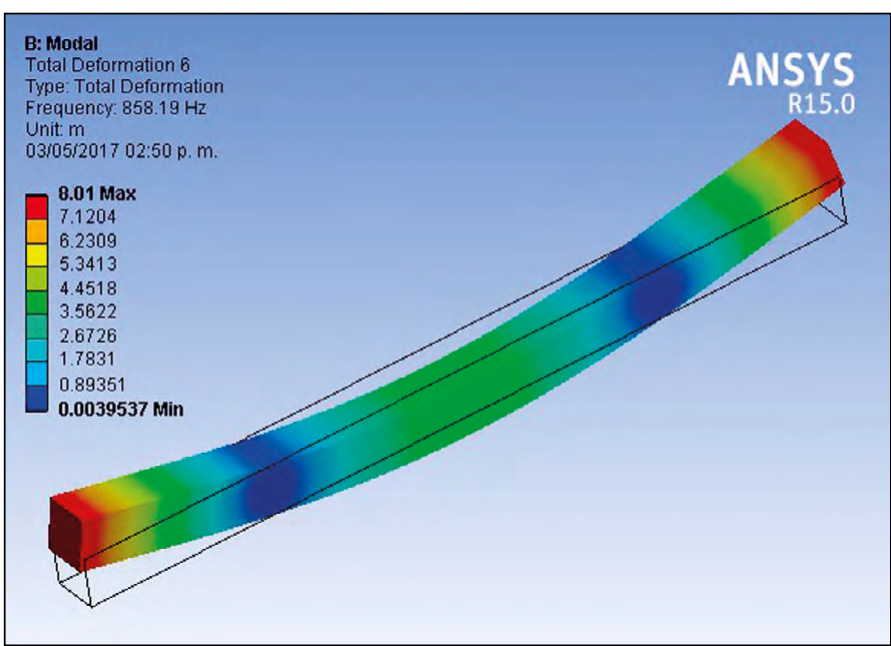

Figura 3. Deformación de una probeta de C. odorata en el primer modo de vibración.

Elaboración propia.

DISCUSIÓN

Las magnitudes de la densidad cubrieron un intervalo de $544 \mathrm{~kg} \mathrm{~m}^{-3}$, con un mínimo para Spathodea campanulata de $357 \mathrm{~kg} \mathrm{~m}-3$ y un máximo para Acacia farnesiana de $901 \mathrm{~kg} \mathrm{~m}^{-3}$. Los valores del módulo dinámico experimental comprendieron un intervalo de $10312 \mathrm{MN} \mathrm{m}^{-2}$, con un mínimo de $3765 \mathrm{MN} \mathrm{m}^{-2}$ para S. campanulata y un máximo de $14077 \mathrm{MN} \mathrm{m}^{-2}$ para Platymiscium dimorphandrum. Asimismo, los valores de la frecuencia experimental cubrieron un rango de $291 \mathrm{~Hz}$, con un mínimo para S. campanulata de $655 \mathrm{~Hz}$, y un máximo de $987 \mathrm{~Hz}$ para Cupressus lindleyi.

Para las frecuencias experimental y numérica, los valores del sesgo y de la curtosis estandarizados se situaron al interior del intervalo $-2,+2$. Conjuntamente, el valor $P=0.4197$ de la prueba de conformidad de varianzas verificó igualmente que no existe una diferencia estadísticamente significativa entre las desviaciones estándar de las muestras observadas. Asimismo, el valor $P=0.9308$ del análisis de varianza confirmó que no existe una diferencia estadísticamente significativa entre las medias de las dos variables. En consecuencia, desde el punto de vista estadístico, las magnitudes de la frecuencia predichas numéricamente equivalen a las determinadas experimentalmente.

Sin embargo, los resultados de la tabla 1 sugieren, para cada especie, diferencias aritméticas entre las frecuencias experimentales y numéricas. En efecto, se puede observar una diferencia máxima entre $f_{\text {exp }}$ y $f_{\text {MEF }}$ de $14.1 \%$ para A. farnesiana, especie que presentó la máxima densidad. Con todo, esta diferencia no es proporcional en función de la densidad de la madera. En efecto, la diferencia mínima entre frecuencias es de $1.7 \%$ y corresponde a Cedrela odorata con densidad de $566 \mathrm{~kg} \mathrm{~m}^{-3}$. Esta tendencia se indica en la figura 4 que muestra la correlación de la frecuencia experimental en función de la densidad de la madera y su coeficiente de determinación. En las figuras 4 y 5 la leyenda de los puntos corresponde a la numeración de las especies de la tabla 1.

En efecto, el coeficiente de determinación de la regresión $f_{\text {exp }}=f\left(\rho_{\mathrm{CH}}\right)$ es $R^{2}=0.71$, valor aceptable de acuerdo con el paradigma vigente para ciencias y tecnología de la madera, el cual considera a la densidad como predictor de sus características elásticas, en este caso, del módulo dinámico (Niklas \& Spatz, 2010). Sin embargo, no se observa una correspondencia inequívoca entre módulo dinámico y densidad de la madera, ambos experimentales.

Como consecuencia, los resultados numéricos que utilizan estos datos como parámetros materiales en el modelo resultan en una variación correspondiente $\mathrm{y} / \mathrm{o}$ similar. Este resultado se explica en la figura 5 que presenta la correlación de la frecuencia experimental $\left(f_{\exp }\right)$ en función de la frecuencia numérica $\left(f_{M E F}\right)$, con un coeficiente de determinación aceptable, pero menor $\left(R^{2}=0.66\right)$.

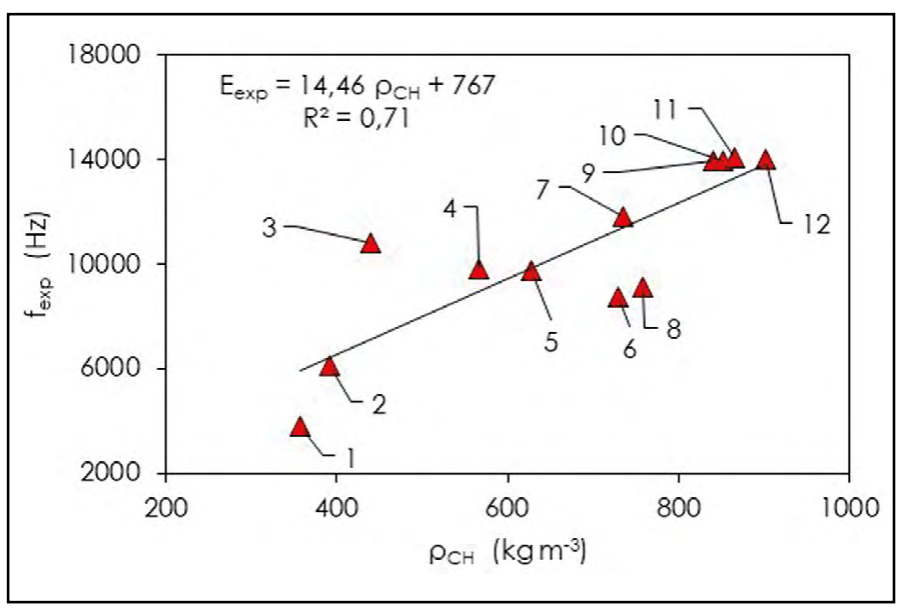

Figura 4. Dispersión de la frecuencia experimental ( $f_{\exp }$ ) en función de la densidad de la madera $\left(\rho_{C H}\right)$, su regresión y coeficiente de determinación $\left(R^{2}\right)$.

Elaboración propia. 


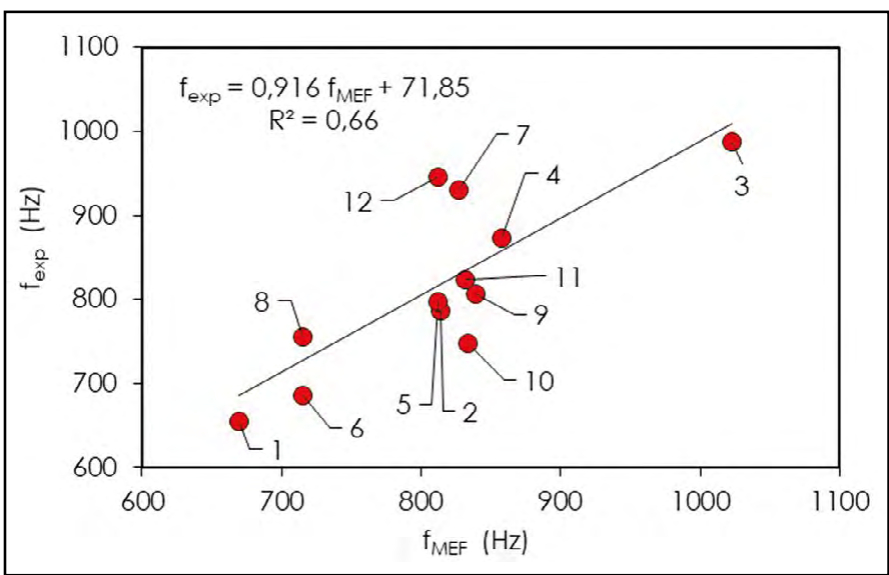

Figura 5. Dispersión de la frecuencia experimental ( $f_{\text {exp }}$ ) en función de la frecuencia numérica $\left(f_{M E F}\right)$, su regresión y coeficiente de determinación $\left(R^{2}\right)$.

Elaboración propia.

La calidad de un procedimiento de caracterización mecánica de la madera está delimitada principalmente por dos factores: el primero es la precisión del parámetro medido para explicar el parámetro dependiente, esta capacidad es cuantificada por medio del coeficiente de determinación $\left(R^{2}\right)$ derivado del análisis de regresión, y el segundo factor es el error introducido en la predicción del parámetro, el cual puede ser valorado por el coeficiente de variación (CV) de las mediciones. Si el análisis de regresión entre dos parámetros 0 variables se deriva de mediciones realizadas en las mismas condiciones experimentales y con el mismo aparato, el efecto del error de medición y del coeficiente de variación ya está incluido en el valor de $R^{2}$ directamente (Hanhijärvi, Ranta-Maunus, \& Turk, 2005). Retomando este argumento, el coeficiente de determinación entre $f_{\text {exp }}$ versus $\rho_{C H}$ fue de 0.71 (figura 4 ) y en comparación con el coeficiente de determinación entre $f_{\text {MEF }}$ versus $\rho с н$ que fue de 0.66 (figura 5), es decir, una disminución de $7 \%$.

La estrategia de comparar resultados experimentales con numéricos es reportada recientemente por Christoforo et al. (2016), quienes determinaron para Pinus elliottti $\left(570 \mathrm{~kg} \mathrm{~m}^{-3}\right)$ los módulos de elasticidad estáticos de manera experimental y compararon sus resultados con los módulos estimados con el MEF. Estos autores encontraron un coeficiente de determinación entre los dos módulos de 94.5\%. Por su parte, Kandler, Füssl, Serrano y Eberhardsteiner (2015) encontraron coeficientes de correlación entre los módulos dinámicos experimentales y numéricos de 0.88. Por su parte, Lukacevic, Füssl, Griessner y Eberhardsteiner (2014) reportan igualmente que la simulación numérica es capaz de reproducir adecuadamente el comportamiento estructural básico de piezas de madera de tamaño estructural de Picea abies $\left(\rho_{\mathrm{CH}}=390 \mathrm{~kg} \mathrm{~m}^{-3}\right)$ y completa la validación. Sus resultados experimentales y numéricos de resistencia mecánica en flexión resultaron con un coeficiente de determinación de 0.79. Comparando con los resultados de esta investigación (2017), los coeficientes de determinación fueron menores que los reportados en la literatura.

CONCLUSIONES

Se determinó experimentalmente y se verificó por el método del elemento finito la frecuencia natural de barras de madera ensayadas en vibraciones transversales. El modelo fue validado experimentalmente comparando las predicciones del modelo para 12 especies leñosas, con los resultados promedio de 32 especímenes ensayados en vibraciones transversales para cada una de las mismas especies.

Desde el punto de vista estadístico las magnitudes de la frecuencia predichas numéricamente son equivalentes a las determinadas experimentalmente. El uso del módulo dinámico obtenido experimentalmente y empleado en las simulaciones del movimiento de probetas de 12 especies de madera conduce a valores de frecuencia equivalentes a los obtenidos en las pruebas de vibraciones, probando así la eficiencia del modelado numérico para estimar la frecuencia de una pieza de madera. Este corolario es particular para las condiciones experimentales y específicas a este estudio de caso. Para prospecciones más generales se recomienda realizar estudios intensivos para las condiciones de carga y para la configuración de las pruebas correspondientes.

La densidad de la madera predice su módulo dinámico hasta en $71 \%$. La comparación de la predicción del modelo numérico con los resultados experimentales, en términos de la correlación entre la frecuencia numérica y la frecuencia experimental, explica $66 \%$ de esta dependencia. Así, la regresión lineal calculada indica una moderada calidad predictiva del modelo de simulación empleado en la investigación. 
Con todo, los coeficientes de determinación reportados en la bibliografía y citados anteriormente son mayores que los obtenidos en esta investigación. El modelo numérico aquí utilizado parte de que el material con el cual está conformado el volumen puesto en vibración es homogéneo e isotrópico. Esta hipótesis material introducida para simplificar el tratamiento numérico no satisface necesariamente las características materiales de las probetas estudiadas experimentalmente. En efecto, la estructura anatómica de cada una de las especies es diferente y particular, argumento que sugiere para futuras simulaciones similares a las propuestas en esta investigación detallar más el modelo material de la madera.

\section{Agradecimientos}

Los autores desean agradecer al Doctor Juan Zárate Medina, profesor de la Universidad Michoacana de San Nicolás de Hidalgo, por su colaboración en la realización de las pruebas de vibraciones transversales. La investigación estuvo patrocinada por la Coordinación de la Investigación Científica, de la misma universidad.

\section{REFERENCIAS}

- Baño, V., Arriaga, F., Soilán, A., \& Guaita, M. (2011). Prediction of bending load capacity of timber beams using a finite element method simulation of knots and grain deviation. Biosystems Engineering, 109(4), 241-249. doi: 10.1016/j. biosystemseng.2011.05.008

- Christoforo, A. L., Panzera, T. H., Silveira, M, E., Lopes Silva, D. A., Vasconcelos Pinheiro, R., \& Rocco Lahr, F. A. (2016). Numerical evaluation of the bending modulus of elasticity in Pinus elliottii round timber beams. Ciência Florestal, 26(4), 1271-1279. doi: 10.5902/1980509825138

- De Amicis, R., Riggio, M., Girardi, G., \& Piazza, M. (2011). Morphology-based macro-scale finite-element timber models. Computer-Aided Design, 43(1), 72-87. doi: 10.1016/j. cad.2010.09.003

- De Borst, K., Jenkel, C., Montero, C., Colmars, J., Gril, J., Kaliske, M., \& Eberhardsteiner, J. (2013). Mechanical characterization of wood: An integrative approach ranging from nanoscale to structure. Computers and Structures, 127, 53-67. doi: 10.1016/j. compstruc.2012.11.019

- Gaff, M., Gašparik, M., Borůvka, V., \& Babiak, M. (2015). Simulating stresses associated with the bending of wood using a Finite Element M. BioResources, 10(2), 2009-2019. doi: 10.15376/biores.10.2.2009-2019

- García, D. A., Sampaio, R., \& Rosales, M. B. (2016). Eigenproblems in timber structural elements with uncertain properties. Wood Science and Technology, 50(4), 807-832. doi: 10.1007/s00226-016-0810-8

- Gonçalves, R., Trinca, A. J., \& Pellis, B. P. (2014). Elastic constants of wood determined by ultrasound using three geometries of specimens. Wood Science and Technology, 48(2), 269-287. doi: 10.1007/s00226-013-0598-8

- Guitard, D., \& Gachet, C. (2004). Paramètres structuraux et/ ou ultrastructuraux facteurs de la variabilité intra-arbre de
I'anisotropie élastique du bois. Annals of Forest Science, 61 (2), 129-139. doi: 10.1051/forest:2004004

- Gutiérrez Pulido, H., \& De la Vara Salazar, R. (2012). Análisis y diseño de experimentos ( $3^{a}$. ed). México: McGraw-Hill.

- Hackspiel, C., de Borst, K., \& Lukacevic, M. (2014a). A numerical simulation tool for wood grading model development. Wood Science and Technology, 48(3), 633-649. doi: 10.1007/s00226014-0629-0 grading: Model validation and parameters studies. Wood Science and Technology, 48(3), 651-669. doi: 10.1007/s00226014-0630-7

- Hanhijärvi, A., Ranta-Maunus, A., \& Turk, G. (2005). Potential of strength grading of timber with combined measurement techniques. Report of the Combigrade-project-phase 1 . Finland: VTT Publications. Recuperado de http://www.vtt.fi/ inf/pdf/publications/2005/P568.pdf

- Hofstetter, K., Hellmich, C., \& Eberhardsteiner, J. (2004). Hierarchical organization of wood revisited in the framework of continuum micromechanics. En P. Neittaanmäki et al. (Eds.), European Congress on Computational Methods in Applied Sciences and Engineering (pp. 1-21). Jyväskylä: University of Jyväskylä. Recuperado de https://www.researchgate.net/ publication/267236732_Hierarchical_organization_of_wood_ revisited_in_the_framework_of_continuum_micromechanics

- Hwang, Y. F., \& Suzuki, H. (2016). A finite-element analysis on the free vibration of Japanese drum wood barrels under material property uncertainty. Acoustical Science and Technology, 37(3), 115-122. doi: 10.1250/ast.37.115

- International Organization for Standardization. (2012). ISO 3129:2012. Wood. Sampling methods and general requirements for physical and mechanical testing of small clear wood specimens. Geneva: International Organization 
for Standardization. Recuperado de https://www.iso.org/ standard/52489.html

- International Organization for Standardization. (2014a). ISO 13061-1:2014. Wood. Determination of moisture content for physical and mechanical tests. Geneva: International Organization for Standardization. Recuperado de https:// www.iso.org/standard/60063.html

- International Organization for Standardization. (2014b). ISO 13061-2:2014. Wood. Determination of density for physical and mechanical tests. Geneva: International Organization for Standardization. Recuperado de https://www.iso.org/ standard/60064.html

- Kandler, G., Füssl, J., \& Eberhardsteiner, J. (2015). Stochastic finite element approaches for wood-based products: Theoretical framework and review of methods. Wood Science and Technology, 49(5), 1055-1097. doi: 10.1007/s00226-0150737-5

- Kandler, G., Füssl, J., Serrano, E., \& Eberhardsteiner, J. (2015). Effective stiffness prediction of GLT beams based on stiffness distributions of individual lamellas. Wood Science and Technology, 49(6), 1101-1121. doi: 10.1007/s00226-015-0745-5

- Kouroussis, G., Ben Fekih, L., \& Descamps, T. (2017). Assessment of timber element mechanical properties using experimental modal analysis. Construction and Building Materials, 134, 254261. doi: 10.1016/j.conbuildmat.2016.12.081

- Lukacevic, M., Füssl, J., Griessner, M., \& Eberhardsteiner, J. (2014). Performance assessment of a numerical simulation tool for wooden boards with knots by means of full-field deformation measurements: Performance assessment of a numerical simulation tool for wooden boards. Strain, 50(4), 301-317. doi: $10.1111 /$ str.12093

- Machek, L., Militz, H., \& Sierra-Alvarez, R. (2001). The use of an acoustic technique to assess wood decay in laboratory soilbed tests. Wood Science and Technology, 34 (6), 467-472. doi: $10.1007 / \mathrm{s} 002260000070$

- Mackerle, J. (2005). Finite element analyses in wood research: A bibliography. Wood Science and Technology, 39(7), 579600. doi: 10.1007/s00226-005-0026-9
- Niklas, K. J., \& Spatz, H. C. (2010). Worldwide correlations of mechanical properties and green wood density. American Journal of Botany, 97(10), 1587-1594. doi: 10.3732/ajb.1000150

- Ormarsson, S., Dahlblom, O., \& Johansson, M. (2009). Finite element study of growth stress formation in wood and related distortion of sawn timber. Wood Science and Technology, 43(5-6), 387-403. doi: 10.1007/s00226-008-0209-2

- Silva Guzmán, J. A., Fuentes Talavera, F. J., Rodríguez Anda, R., Torres Andrade, P. A., Lomelí Ramírez, M. A., Ramos Quirarte, J.,... \& Richter, H. G. (2010). Fichas de propiedades tecnológicas y usos de maderas nativas de México e importadas. México: Comisión Nacional Forestal.

- Sotomayor-Castellanos, J. R. (2015). Banco FITECMA de características físico-mecánicas de maderas mexicanas (65 p.). Morelia: Universidad Michoacana de San Nicolás de Hidalgo. Recuperado de https://www.researchgate.net/ publication/276841418_Banco_FITECMA_de_caracteristicas fisico-mecanicas_de_maderas_Mexicanas

- Sotomayor-Castellanos, J. R., \& López-Garza, V. (2016). Verificación por el método del elemento finito del comportamiento mecánico de la madera en flexión estática. Investigación e Ingeniería de la Madera, 12(3), 4-54. Recuperado de https://www.researchgate.net/ publication/311651698_Verificacion_por_el_metodo_del_ elemento_finito_del_comportamiento_mecanico_de_la_ madera_en_flexion_estatica

- Tamarit Urias, J. C., \& López Torres, J. L. (2007). Xilotecnología de los principales árboles tropicales de México. México: Instituto Nacional de Investigaciones Forestales, Agrícolas y Pecuarias campo experimental San Martinito.

- Vasic, S., Smith, I., \& Landis, E. (2005). Finite element techniques and models for wood fracture mechanics. Wood Science and Technology, 39(1), 3-17. doi: 10.1007/s00226-004-0255-3

- Villaseñor-Aguilar, J. M., \& Sotomayor-Castellanos, J. R. (2015). Caracterización dinámica de la madera de Fraxinus americana y Fraxinus uhdei. Revista de Aplicación Científica y Técnica, 1 (1), 43-53. Recuperado de http://www.ecorfan. org/spain/researchjournals/Aplicacion_Cientifica_y_Tecnica/ vol1num 1/Aplicacion-Cientifica-y-Tecnica--50-60.pdf

- Weaver, W., Timoshenko, S., \& Young, D. H. (1990). Vibration problems in engineering (5a. ed.). New York: Wiley. 